DOI: $10.1002 /(($ please add manuscript number $))$

Article type: Communication

\title{
Cyclodextrin films with fast solvent transport and shape-selective permeability
}

Luis Francisco Villalobos, Tiefan Huang and Klaus-Viktor Peinemann*

L. F. Villalobos, T. Huang, Prof. K.-V. Peinemann

King Abdullah University of Science and Technology (KAUST), Advanced Membranes and Porous Materials Center, Thuwal 23955-6900, Kingdom of Saudi Arabia

E-mail: klausviktor.peinemann@kaust.edu.sa

Keywords: cyclodextrin, membrane, nanofiltration, free-volume, interfacial polymerisation

\begin{abstract}
We describe the molecular-level design of a new type of filtration membrane made of crosslinked cyclodextrins - inexpensive macrocycles of glucose, shaped like hollow truncated cones. The channel-like cavities of cyclodextrins spawn numerous paths of defined aperture in the separation layer that can effectively discriminate between molecules. The transport of molecules through these membranes is highly shape-sensitive. In addition, the presence of hydrophobic (cavity) and hydrophilic (ester-crosslinked outer part) domains in these films results in high permeances for both polar and non-polar solvents.
\end{abstract}

\section{Main Text}

Energy-intensive molecular separations often impose the heaviest economical burden and the biggest environmental footprint of industrial processes. However, the energetic demands of these separations can be reduced considerably by using high-performance membranes rather than a phase change process. ${ }^{[1]}$ Energy-efficient separations require highly permeable membranes with rigorous selective properties in a targeted size range. ${ }^{[2]}$ The performance of these membranes can be maximised by choosing the appropriate material and controlling the porosity and thickness of the selective layer. 
Unlike rigid microporous materials, such as metal-organic frameworks, zeolites, porous coordination polymers, and covalent organic frameworks that have defined cavity shapes and sizes, amorphous polymers contain a wide range of cavity sizes and topologies. ${ }^{[3]}$ Nevertheless, the ease of processing and economical cost of these polymers has positioned them as the workhorse materials of the membrane industry. Their performance in smallmolecule separations is governed by the size, distribution, and connectivity of the disordered voids - free volume - that occur in them naturally. Ideally, the free volume of polymeric materials should be permanent and interconnected and have a narrow aperture-size distribution; however, controlling these properties has proven to be challenging. ${ }^{[4,5]}$ Successful strategies to increase the free volume of and create more interconnected voids within these membranes have focused on preventing the efficient packing of polymer chains in the solid state by adding filler particles as "nanospacers", ${ }^{[6]}$ using block copolymer selfassembly, ${ }^{[7]}$ and synthesising polymers of intrinsic microporosity. ${ }^{[8]}$ Efforts have also been made to control the size distribution of the free-volume elements (i.e., cavities) by inducing thermally driven segment rearrangements. ${ }^{[5]}$ We propose an approach that uses an intrinsically porous macrocycle as the main building block of the membrane's selective layer to increase the free-volume and to achieve certain control over the size and shape of its free-volume elements. Each macrocycle supplies the matrix with a free-volume element of fixed dimensions. Among the available intrinsically porous building blocks to choose from, ${ }^{[9]}$ cyclodextrins are particularly attractive because their large-scale production from starch is sustainable ${ }^{[10]}$ they are non-toxic ${ }^{[11]}$ and they have already proven to be useful agents for a wide variety of separations. ${ }^{[12]}$

The preparation of cyclodextrin-based crosslinked polymers has been going on for more than 50 years. ${ }^{[13,14]}$ Surprisingly, the fabrication of a membrane made entirely of cyclodextrins has remained elusive. Rölling and colleagues' attempt to do this by radical polymerisation of acrylated cyclodextrins yielded membranes that were too brittle for use. ${ }^{[15]}$ The flexibility of 
the membranes was rescued by copolymerising cyclodextrins with flexible polymers, but this substantially decreased the concentration of cyclodextrin in the active layer, diluting their effect. Yue et al. developed an ingenious approach with $\alpha-C D$ as a directing agent in the formation of single-layer ionic organic-inorganic 2-D frameworks. ${ }^{[16]}$ The cavity of the $\alpha$-CDs was used as a cap for the "stick" cationic molecules used to build the frameworks. Such layers, when assembled into membranes by vacuum filtration, showed a precise size-selectiveness in the ultrafiltration range. To date, cyclodextrins have been incorporated into membranes as fillers, as part of a membrane-forming polymer, and as surface modifiers (see Supplementary Information, Table S1). This letter highlights how the careful choice of fabrication conditions enables the manufacture of thin, defect-free, solvent and mechanically stable membranes made entirely of crosslinked cyclodextrin molecules.

We formed thin films of cyclodextrin by interfacial polymerisation, a technique used widely in the membrane industry to make thin-film-composite polyamide membranes for water desalination and nanofiltration. The commercially most successful reverse osmosis membrane for water desalination is made by an interfacial reaction using an aqueous amine solution and a solution of trimesoyl chloride in a hydrocarbon. Here, we used a hydrocarbon-based terephtaloyl chloride (TC) solution and cyclodextrins in the aqueous phase instead of the conventional diamines (e.g., m-phenylenediamine, p-phenylenediamine, piperazine). This approach presents several challenges: (i) cyclodextrins are much larger and bulkier than diamines, ${ }^{[17]}$ (ii) the hydroxyl $(\mathrm{OH})$ groups of cyclodextrins are less reactive than amines, and have different reactivities among them, ${ }^{[18]}$ and (iii) cyclodextrins tend to agglomerate in aqueous solutions. ${ }^{[19,20]}$ Nevertheless, by optimising preparation conditions, we produced continuous $\beta$-CD films on top of a commercial polyacrylonitrile (PAN) porous support (Figure 1). 


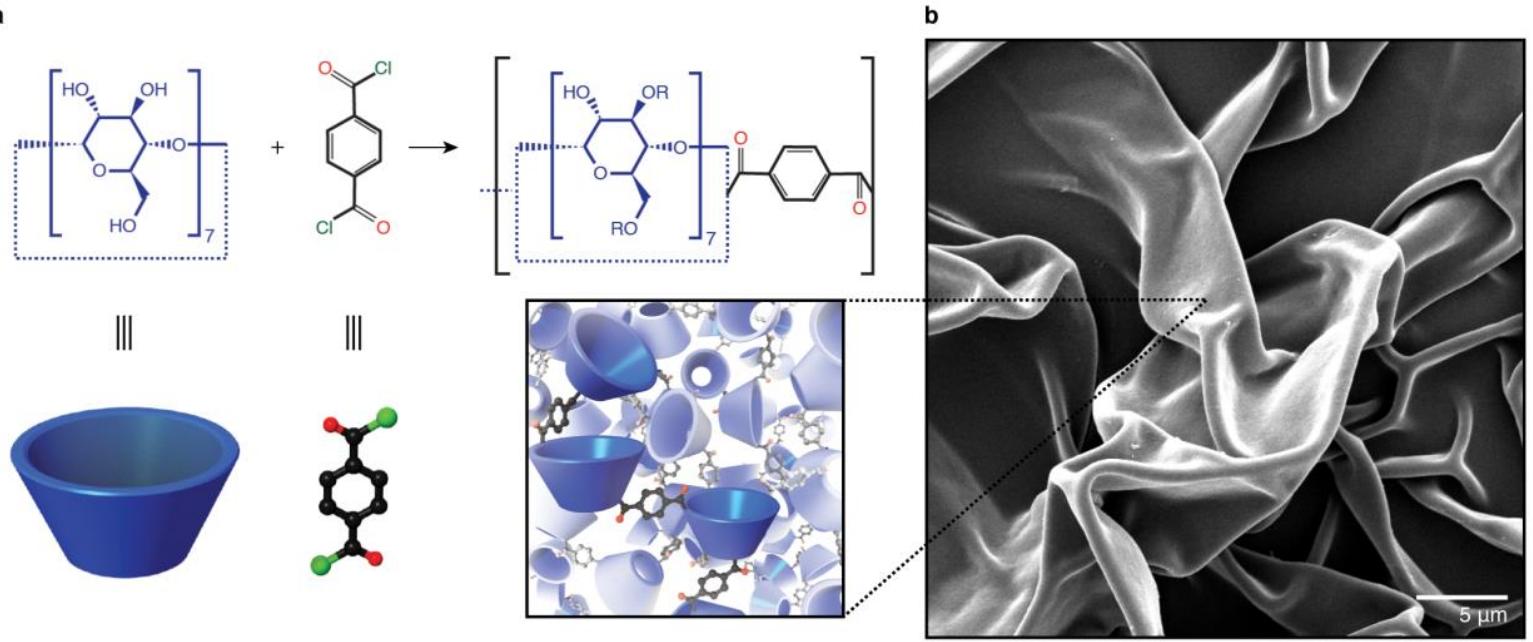

Figure 1. $\beta$-CD films made by interfacial polymerisation on top of PAN porous supports. a) Synthesis of the films from $\beta$-CD (dissolved in the aqueous phase) and terephthaloyl chloride (dissolved in the organic phase). b) SEM image of a $\beta$-CD film made with $6.5 \%(\mathrm{w} / \mathrm{v}) \beta-\mathrm{CD}$ in a $1 \mathrm{M} \mathrm{NaOH}$ solution (aqueous phase), $1 \%(\mathrm{w} / \mathrm{v}) \mathrm{TC}$ in hexane (organic phase), for $10 \mathrm{~min}$ reaction time.

The chemical reaction rate and the transfer of reactants to the polymerisation zone are important factors during interfacial polymerisation, ${ }^{[21]}$ as they can substantially influence the morphology of the resulting film. ${ }^{[22,23]}$ When dealing with nanometer-sized molecules, such as cyclodextrins, diffusion is slow and could be the limiting step if precautions are not taken. To obviate this limitation and to promote a constant and homogeneous availability of cyclodextrins in the reaction zone, we used high concentrations of cyclodextrins and a hydrophilic support with high surface porosity and small pore-size distribution. We found that the use of lower concentrations of cyclodextrins or hydrophobic supports with more surface pore heterogeneity led to defective films (see Supplementary Information, Section B).

A key parameter in the fabrication of cyclodextrin membranes was the $\mathrm{pH}$ of the aqueous phase, which was controlled by addition of sodium hydroxide $(\mathrm{NaOH})$. Hydroxyl groups of $\beta$ $\mathrm{CD}$ start to slowly deprotonate above $\mathrm{pH} 12.0$ and do so more rapidly above $\mathrm{pH} 13.0 .^{[24]}$ Ionisation of $\mathrm{OH}$ groups at high $\mathrm{pHs}$ avoids aggregation of cyclodextrins, ${ }^{[20]}$ forms the more reactive alkoxide ions ${ }^{[18,21]}$ and increases their solubility in water. Each glucopyranose unit in a cyclodextrin molecule has two secondary hydroxyl groups (wide face) and one primary 
hydroxyl group (narrow face). The primary $\mathrm{OH}$ groups are more reactive, however the secondary ones deprotonate easier. At sufficiently high $\mathrm{pHs}$, the acid chloride attacks the hydroxyl groups of the cyclodextrins indiscriminately. ${ }^{[18]}$ For these reasons it was essential to use $\mathrm{NaOH}$ concentrations of $0.1 \mathrm{M}$ or more to form the $\beta$-CD films.

Surprisingly, the morphology of the film was considerably affected by the concentration of $\mathrm{NaOH}$ used: $0.1-0.8 \mathrm{M}$ produced flat, smooth films and 1.0 $\mathrm{M}$ or higher produced crumpled films (Figure S3). We believe that two processes are occurring at the same time-formation and hydrolysis of ester bonds - and that the balance between them determines the morphology of the resulting film. The ester linkages, formed during interfacial polymerisation, can be readily hydrolysed by hydroxide ions. For example, an aqueous solution of $\mathrm{pH} 13.0$ or higher was sufficient to immediately destroy a fully formed $\beta$-CD film, while a solution of $\mathrm{pH} 11.0$ decomposed it slowly. Although the alkaline conditions used in the aqueous phase of the interfacial polymerisation have the potential to destroy the ester links that hold the film together, the low aqueous solubility of the acid chloride prevents the film from forming in the aqueous phase. Hydrolysis of ester bonds can only occur along the bottom of the film and the rate is proportional to the concentration of hydroxyl ions in the aqueous solution stored inside the pores of the PAN support. Slow hydrolysis rates allow for the formation of a continuous highly crosslinked $\beta-\mathrm{CD}$ film along the top of the support. Once formed, the film hinders further diffusion of cyclodextrins to the reaction zone. Alternatively, high hydrolysis rates prevent the formation of a continuous $\beta$-CD film on the support, and instead it forms away from the surface, in some part of the membrane, generating a crumpled structure. The top part of crumpled films suffers less hydrolysis of ester bonds because it is further away of the aqueous phase during their fabrication. Consequently, crumpled films must have a higher crosslinking degree at their top part. X-ray photoelectron spectroscopy (XPS), and FTIR analysis of the top and bottom part of an isolated crumple $\beta$-CD film supported this hypothesis. Both, XPS (Figure 2c-2f) and FTIR (Figure S11) analysis, showed clear 
differences between the bottom and top part of the same $\beta$-CD film indicating an asymmetry in its structure. Using data from the O1s spectra (Figure $2 \mathrm{~d}$ and $2 \mathrm{e}$ ) it is possible to estimate the number of TC molecules per $\beta$-CD (refer to Supplementary Information, Section C.2 for details on the estimation). For the bottom part it ranges from 1.6 to 4.1 , and for the top part from 2.9 to 7.4. A higher number of $\mathrm{TC}$ units per $\beta-\mathrm{CD}$ is an indication of a higher crosslinking degree. Before interfacial polymerization occurs, some of the hydroxyl ions present in the aqueous phase are consumed to ionise $\beta-\mathrm{CD}$ molecules and to partially hydrolyse the PAN support. ${ }^{[25]}$ For this reason $\mathrm{NaOH}$ concentrations of $1 \mathrm{M}$ or more were needed for sufficient presence of hydroxyl ions inside the pores of the support to hydrolyse the $\beta$-CD film at the rate necessary to achieve the desired crumpled morphology. 
a

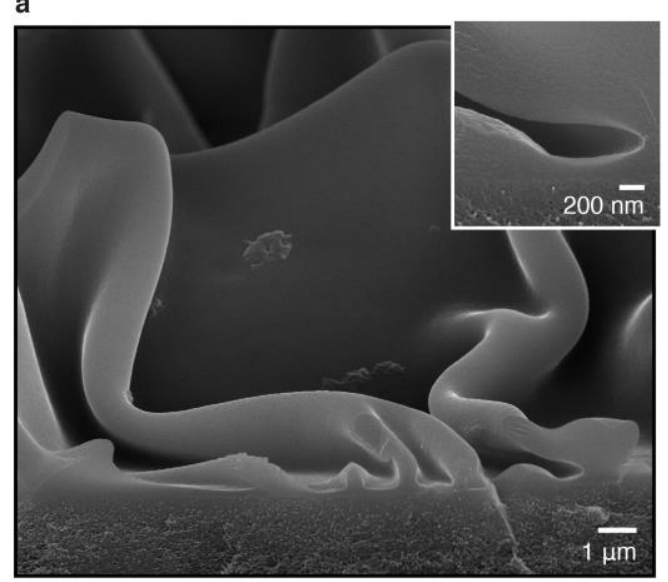

c

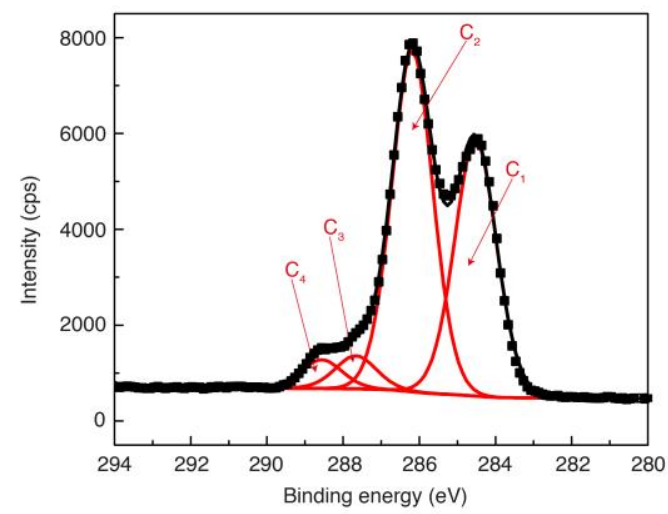

e

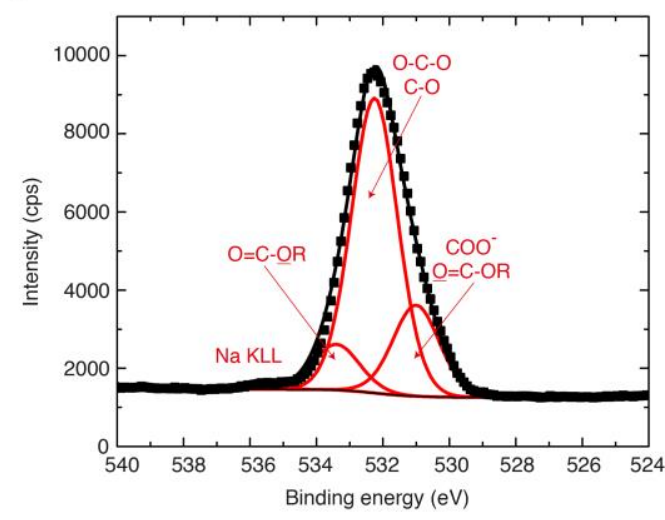

b

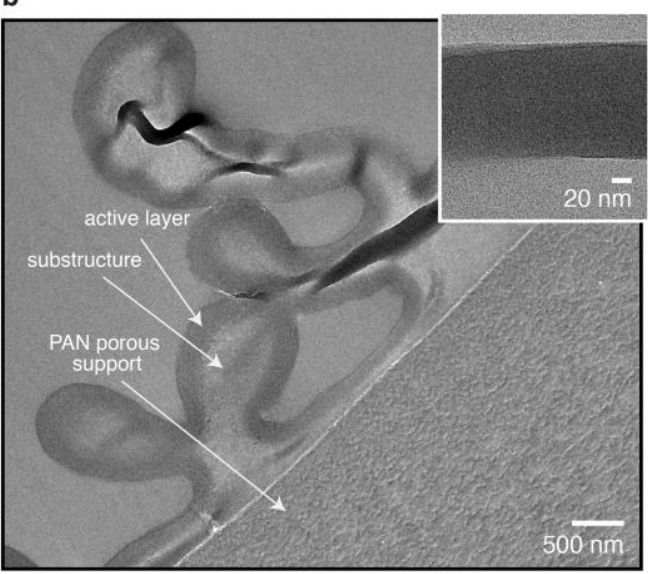

d

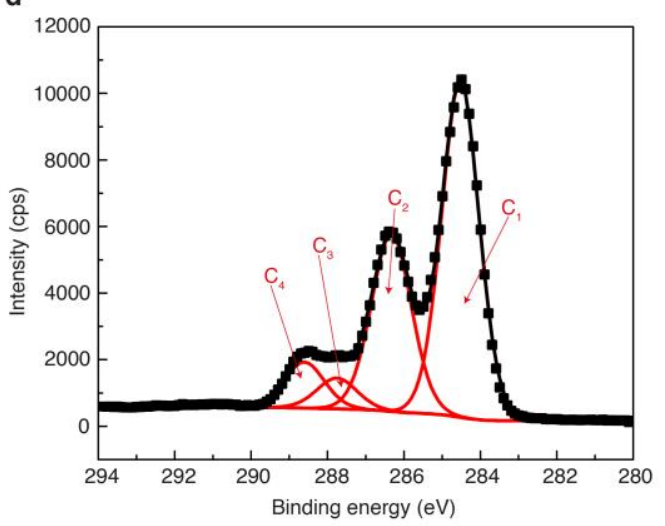

f

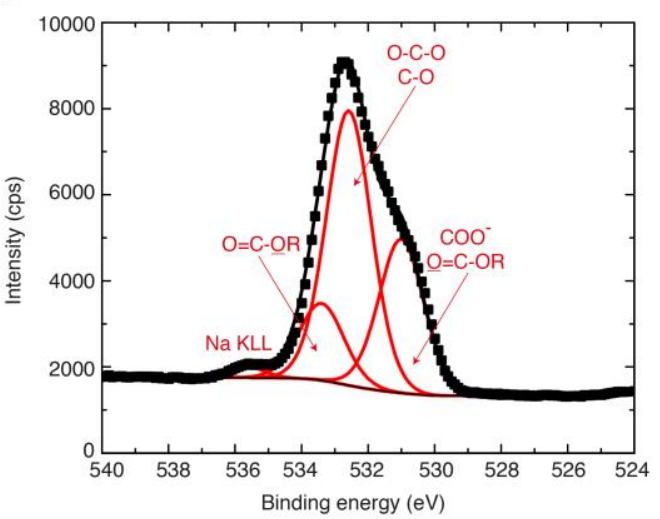

Figure 2. Cross-section and internal structure of a $\beta-\mathrm{CD}$ membrane. The membrane was prepared by interfacial polymerisation of $\beta-\mathrm{CD}(6.5 \%, \mathrm{w} / \mathrm{v})$ in a $1 \mathrm{M} \mathrm{NaOH}$ aqueous solution with TC $(1 \%, \mathrm{w} / \mathrm{v})$ in hexane. a) Cross-sectional SEM. Inset: enlargement of an area encompassing the interface of the $\beta$-CD film with the PAN support. b) TEM image of the $\beta$ CD membrane's cross-section. Inset: enlargement of an area encompassing the active layer of the $\beta$-CD film (thickness of $180 \pm 55 \mathrm{~nm}$ ); the higher contrast of the active layer is because the material is denser in that area. c), d), e), and f) Photoelectron spectra of the crumpled $\beta$ $\mathrm{CD}$ isolated from the PAN support and placed on top a silicon wafer. c) C1s of bottom part. d) $\mathrm{C} 1 \mathrm{~s}$ of top part. e) $\mathrm{O} 1 \mathrm{~s}$ of bottom part. f) $\mathrm{O} 1 \mathrm{~s}$ of top part. The groups that could be attributed to each $\mathrm{C}$ peak are $\mathrm{C}-\mathrm{C}, \mathrm{C}=\mathrm{C}$, and $\mathrm{C}-\mathrm{H}$ for $\mathrm{C}_{1}, \mathrm{C}-\mathrm{O}$, and $\mathrm{C} \equiv \mathrm{N}$ for $\mathrm{C}_{2}, \mathrm{O}-\mathrm{C}-\mathrm{O}$ for $\mathrm{C}_{3}$, and $\mathrm{O}=\mathrm{C}-\mathrm{O}$ for $\mathrm{C}_{4}$. 
The internal structure of the $\beta$-CD crumpled films is very interesting. Scanning electron microscopy (SEM) revealed a cyclodextrin film with variable thickness in the micrometer range (Figure $2 \mathrm{a}$ and $3 \mathrm{~d}$ ). Contrary to what was expected from membranes with such a thick active layer, the transport of molecules through them was very fast-water and methanol permeances of up to 20 and $9.41 \mathrm{~m}^{-2} \mathrm{~h}^{-1} \mathrm{bar}^{-1}$, respectively were obtained. Further analysis of a cross-section of the $\beta$-CD film using transmission electron microscopy (TEM) showed that it comprises a dense layer of $180 \pm 55 \mathrm{~nm}$ and a less dense substructure (Figure $2 \mathrm{~b}$ ). The lower density substructure may be the result of a greater degree of hydrolysis of ester crosslinks during its formation. On the contrary, flat $\beta-\mathrm{CD}$ films presented a uniform density throughout; no difference in contrast was observed in their TEM images (Figure S4b). Crumpled films showed up to 47 times higher solvent permeances than did flat films (Figure 3a) partly because of the larger surface area of the crumpled films but also because of the greater number of hydrolysed ester linkages in the film. 

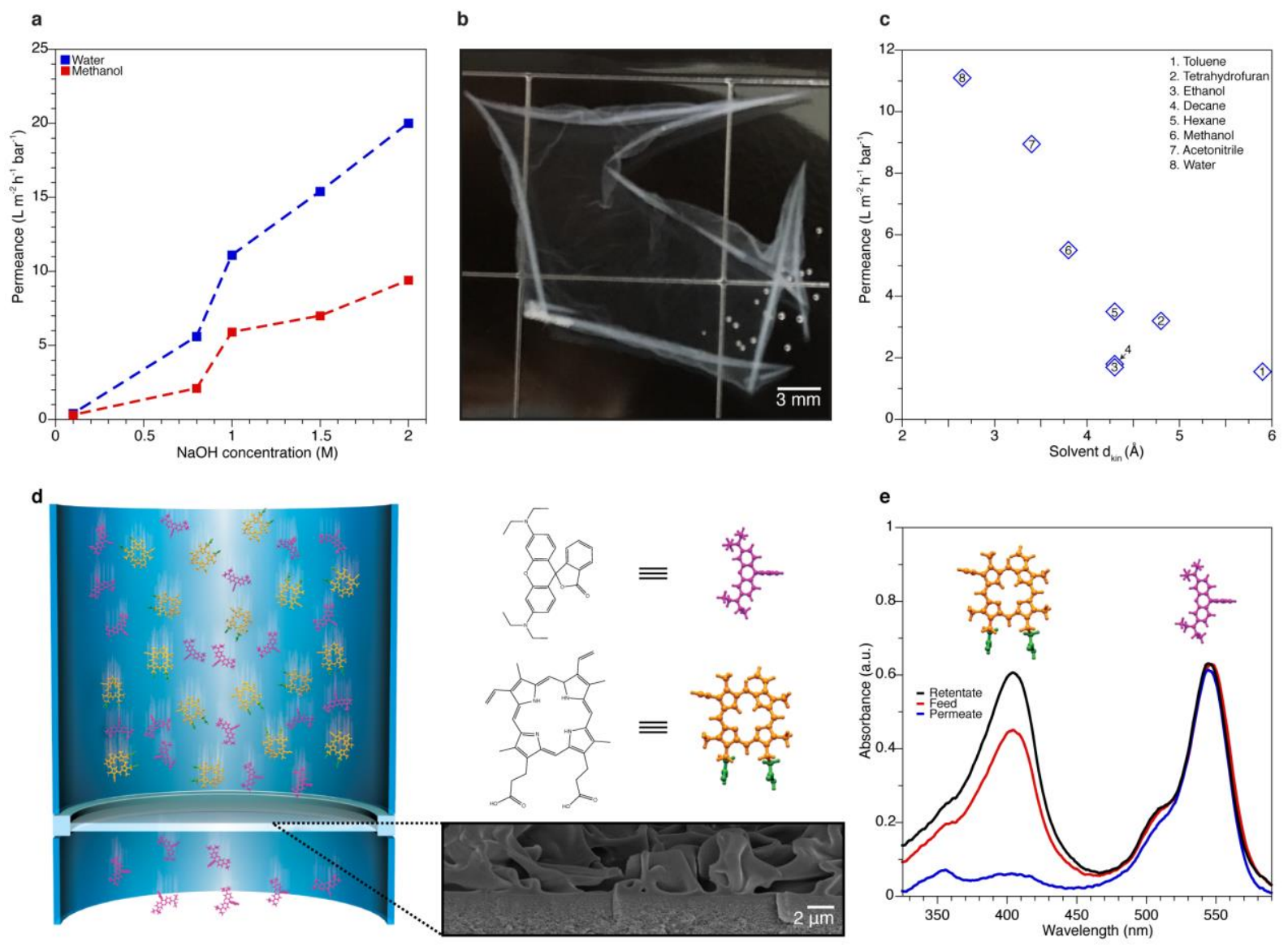

Figure 3. Performance of $\beta-C D$ membranes. a) Pure solvent (water and methanol) permeances of five $\beta$-CD membranes prepared using different $\mathrm{NaOH}$ concentrations in the aqueous phase. The rest of the preparation conditions were the same: $6.5 \%(\mathrm{w} / \mathrm{v}) \beta-\mathrm{CD}$ in aqueous phase, $1 \%(\mathrm{w} / \mathrm{v}) \mathrm{TC}$ in hexane, for $10 \mathrm{~min}$ reaction time. Membranes prepared with 0.1 and $0.8 \mathrm{M} \mathrm{NaOH}$ were flat and smooth, while higher $\mathrm{NaOH}$ concentrations produced crumpled films. b) Photograph of an isolated $\beta$-CD film floating in water. c) Pure solvent permeances for eight solvents through the crumpled $\beta-C D$ membrane prepared using $1 \mathrm{M}$ $\mathrm{NaOH}$ aqueous solution. d) Schematic showing how a $\beta$-CD membrane separates molecules based on their shape. Cross-section SEM image corresponds to a $\beta$-CD membrane prepared using $2 \mathrm{M} \mathrm{NaOH}$ aqueous solution. e) UV-vis absorption spectra of a methanol solution with PPIX (orange molecule) and RB (pink molecule) to evidence the separation performance of the $\beta$-CD membrane.

Molecules can travel either through the cyclodextrin cavity or through the space in between cyclodextrin molecules comprising crosslinker molecules covalently attached to one or two cyclodextrins, carboxylic acid groups produced as a result of ester hydrolysis, and unreacted primary and secondary hydroxyl groups. A greater degree of hydrolysis benefits the transport of molecules through the $\beta$-CD film in two ways: more open pathways in between cyclodextrin molecules and easier access to cyclodextrin's cavity. Using conditions that 
favour ester hydrolysis during fabrication can considerably increase permeance without changing the morphology of the $\beta$-CD film. For example, two flat films prepared with either $0.1 \mathrm{M}$ or $0.8 \mathrm{M} \mathrm{NaOH}$ solution caused permeances differing by an order of magnitude (Figure 3a). An XPS analysis performed to estimate the number of TC units per $\beta$-CD and the degree of ester hydrolysis of each of the $\beta$-CD films is available in the Supplementary Information, Section C.2. Flat $\beta$-CD membranes were outperformed by crumpled membranes, and among them, the one prepared using a $2 \mathrm{M} \mathrm{NaOH}$ solution showed the highest permeance (Figure 3a). However, this membrane contained extensive hydrolysed ester linkages, rendering it unstable at high pressures: It collapsed at pressures above 5 bar. Decreasing the $\mathrm{NaOH}$ concentration to $1 \mathrm{M}$ produced a high-permeance crumpled $\beta-\mathrm{CD}$ film that could withstand pressures of more than 20 bar. SEM images of a $\beta$-CD film after operating for more than 10 hours at 20 bar showed no change in its structure, neither collapse of the crumples (Figure S8c and S8d). However, a decrease in permeance was observed during long-term operation. The methanol permeance dropped around $60 \%$ when using either 4 bar (Figure S8a, membrane tested for 50 hours), or 20 bar (Figure S8b, membrane tested for 13 hours) as the driving force.

The $\beta$-CD film prepared using $1 \mathrm{M} \mathrm{NaOH}$ was isolated by dissolving the PAN support (Figure 3b) and studied it in more detail. The isolated film expanded less than $10 \%$, remained in one piece, and maintained its crumpled structure (Figure S2). It was robust and flexible, showing no visible tearing or fragmentation after several successive aspirations through a pipette (Supplementary Video 1). Moreover, it was thermally stable up to more than $250{ }^{\circ} \mathrm{C}$ in a nitrogen atmosphere (Figure 4a). The number of accessible $\beta$-CD cavities in this film is closely related to the performance of the membrane because molecules can only transport across cyclodextrins with an uncovered cavity. A conservative approximation using ferrocene methanol as a model adsorbate revealed $98 \pm 8 \%$ of cavities were accessible in the film (Figure S12). Moreover, $\mathrm{CO}_{2}$ adsorption experiments at $273 \mathrm{~K}$ revealed a characteristically steep uptake at low relative pressures, indicating a high-degree of microporosity (Figure $4 \mathrm{~b}$ ). 
Non-local density functional theory (NLDFT) calculations applied to the isotherm indicated that the majority of micropores in the $\beta-C D$ film had an aperture of $0.5-0.6 \mathrm{~nm}$ and $0.8 \mathrm{~nm}$ (Figure 4c), which is consistent with that reported for the narrow $(0.61 \mathrm{~nm})$ and wide $(0.79$ $\mathrm{nm}$ ) cavity apertures of $\beta-\mathrm{CD}^{[17]}$ (Figure $\mathrm{S} 14$ ). The surface area of the isolated $\beta-\mathrm{CD}$ film was calculated at $160 \pm 4 \mathrm{~m}^{2} \mathrm{~g}^{-1}$ with the Langmuir model. This value is comparable to results from Jimenez-Solomon et al., who prepared microporous polyarylate nanofilms and reported a Langmuir surface area of $79-90 \mathrm{~m}^{2} \mathrm{~g}^{-1}$ for polymers made by interfacial polymerisation and 150-160 $\mathrm{m}^{2} \mathrm{~g}^{-1}$ for those made by monophasic reaction ${ }^{[26]}$ (calculated from $\mathrm{CO}_{2}$ adsorption experiments).
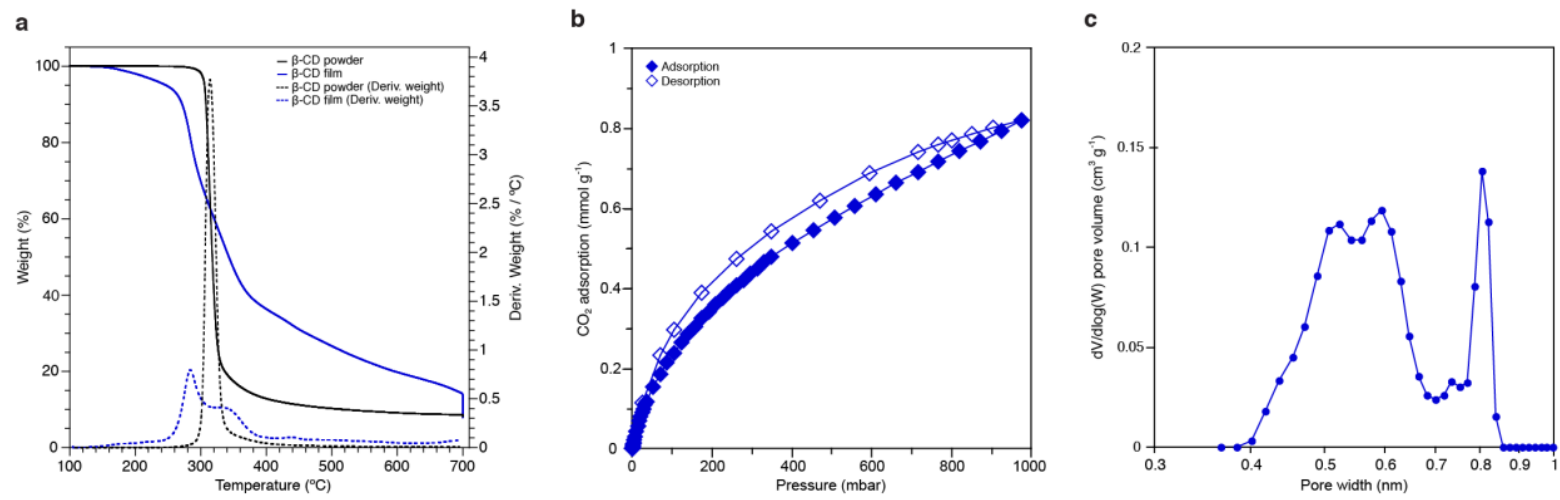

Figure 4. Characterisation of the isolated $\beta-C D$ film. The analysed $\beta-C D$ film was made with $6.5 \%(\mathrm{w} / \mathrm{v}) \beta-\mathrm{CD}$ in a $1 \mathrm{M} \mathrm{NaOH}$ solution (aqueous phase), $1 \%(\mathrm{w} / \mathrm{v}) \mathrm{TC}$ in hexane (organic phase), for $10 \mathrm{~min}$ reaction time. To obtain enough material for the analysis, we isolated $\beta-C D$ films from 20 membranes prepared with the same conditions. a) Thermogravimetric analysis (TGA) curves for $\beta$-CD crosslinked film and pure $\beta$-CD. b) $\mathrm{CO}_{2}$ sorption at $273 \mathrm{~K}$ for the isolated $\beta-C D$ film. c) NLDFT-analysed pore-size distribution based on carbon slit-pore geometry. The cumulative surface area of all the pores up to $1 \mathrm{~nm}$ approximated by NLDFT was $75 \mathrm{~m}^{2} \mathrm{~g}^{-1}$.

The transport of solvent molecules through the $\beta$-CD membranes is determined by the size of the solvent molecules and to a lesser extent by the viscosity and polarity of the solvent. Both polar and nonpolar solvents permeate through the $\beta-\mathrm{CD}$ film at high rates due to the presence of both hydrophilic and hydrophobic domains within it. Data for the successive permeation of several solvents through a crumpled $\beta-\mathrm{CD}$ membrane are available in Figure $3 \mathrm{c}$. Integrity of 
the membrane throughout the experiment was confirmed by testing its rejection of methyl orange (molecular weight $327.3 \mathrm{~g} \mathrm{~mol}^{-1}$, size $0.86 \mathrm{~nm}^{3}$, negative charge) in different solvents. We measured a $90 \%$ or greater rejection for all solvents in which methyl orange was soluble (water, acetonitrile, methanol, ethanol, and tetrahydrofuran). This translates to permeances at least an order of magnitude higher than that of commercially available membranes (see Supplementary Information, Table S2) and comparable to recently published results of stateof-the-art thin-film composite membranes used for solvent resistant nanofiltration (see Supplementary Information, Table S3). Unlike these membranes, however, that rely on a very thin active layer to achieve high permeances, $\beta-\mathrm{CD}$ membranes perform similarly despite having an active layer of at least an order of magnitude thicker (detailed comparison available in Supplementary Information, Section D). $\beta-C D$ membranes have permeances in the same range of thin polyamide, ${ }^{[23]}$ polyarylate $^{[26]}$ or polyelectrolyte bilayer ${ }^{[27]}$ films. And rejections of methyl orange of $91-95 \%$, compared to $96-99 \%$ of methyl orange for polyamide thin films, 97-99\% of crystal violet for polyarylate thin films, and $95-96 \%$ of acid fuchsin for polyelectrolyte bilayer thin films.

The most distinctive feature of the prepared $\beta-\mathrm{CD}$ membranes is their ability to discriminate small molecules based on their shape. Only molecules with a kinetic diameter smaller than the narrow aperture of $\beta$-cyclodextrin's cavity (approx. $0.61 \mathrm{~nm}$ ) were able to transport across it. Additionally, carboxylic acid groups, produced by the hydrolysis of ester linkages or acid chloride groups, hinder negatively charged molecules from entering the cavities. Small, negatively charged molecules that fit inside the cavity of $\beta-\mathrm{CD}$ were rejected, while molecules of similar size but neutral passed freely (Figure S9). Molecules that cannot enter the cavity have to find possible pathways through the available spaces in between the cyclodextrin molecules. For example, two neutral molecules of similar molecular weight, protoporphyrin IX (PPIX; molecular weight $562.7 \mathrm{~g} \mathrm{~mol}^{-1}$, approx. molecular width of $1.5 \mathrm{~nm}^{[28]}$ ) and rhodamine $\mathrm{B}$ base (RB; molecular weight $442.6 \mathrm{~g} \mathrm{~mol}^{-1}$, approx. molecular width of 0.6 
$\mathrm{nm}^{[29]}$ ), can be effectively separated by a $\beta-\mathrm{CD}$ membrane (Figure $3 \mathrm{~d}$ and $3 \mathrm{e}$ ). Permeating a mixture of these two molecules in methanol resulted in a rejection of only $3 \%$ of the cylindrically shaped RB molecules while $90 \%$ of the spherically shaped PPIX were retained. This discriminate shape selectivity has also been verified for larger molecules: A molecule known for its ability to thread cyclodextrin molecules ${ }^{[30]}$ (polyethylene oxide, average molecular weight $1500 \mathrm{~g} \mathrm{~mol}^{-1}$ ) was able to freely cross the $\beta$-CD film while a molecule of similar molecular weight but a shape unconducive with that of the cavity $(\gamma-\mathrm{CD}$, molecular weight $1297.1 \mathrm{~g} \mathrm{~mol}^{-1}$ ) was substantially rejected (Figure S7c, and S7d).

Although we focused on $\beta-\mathrm{CD}$, similar films could also be fabricated with other common cyclodextrins, such as $\alpha$-CD or $\gamma$-CD (Figure S13). By following a well-established process of fabrication combined with the low cost and commercial availability of all the materials used, we demonstrated the feasibility of up-scaling this type of high-performance membrane. We hope this work will inspire the use of cyclodextrins and other cavitands ${ }^{[31]}$ as central building blocks for novel membranes required for tackling challenging molecular separations.

\section{Experimental Section}

Synthesis of cyclodextrin films: Membranes were made using the following conditions unless otherwise is specified: First, a commercial porous PAN membrane (GMT GmbH) was immersed for 30 minutes in a $6.5 \%(\mathrm{w} / \mathrm{v})$ dry $\beta$-cyclodextrin $(\beta-\mathrm{CD}$ hydrate from Acros Organics) $1 \mathrm{M} \mathrm{NaOH}$ aqueous solution. Next, the cyclodextrin loaded PAN support was pressed with a rubber roller to remove excess solution from the surface and mounted in a Teflon frame. Then, the surface of the PAN support was exposed to a $1 \%$ (w/v) TC (SigmaAldrich) in hexane (Sigma-Aldrich) solution for 10 minutes. Finally, the membrane was washed with hexane to remove the unreacted TC, dried for at least 12 hours at room temperature, and then rinsed and stored in Milli-Q water. The Teflon frames used for this work produced square membranes of $7 \times 7 \mathrm{~cm}$ that were cut into circles of 1 or 2 inches to fit either the Millipore plastic cell or the Sterlitech stainless steel cell used in the permeation 
experiments. Control experiments were made with no monomer in both aqueous and organic phases, no monomer in the aqueous phase and no monomer in the organic phase. Detailed information of the optimisation of the preparation conditions is available in the Supplementary Information, Section B.

$\alpha-\mathrm{CD}$ and $\gamma$-CD membranes were made with cyclodextrins obtained from Tokyo Chemical Industry.

Characterisation methods. SEM images were obtained using a FEI Nova Nano or a FEI Quanta 200. Cross-section samples were prepared by freeze fracturing the membranes in liquid nitrogen. Samples were coated with a thin film of Ir or $\mathrm{Au} / \mathrm{Pd}$ to reduce charging during imaging. TEM images were obtained using a FEI Titan 80-300 CT operated at $300 \mathrm{kV}$. To prepare cross-section samples for TEM, the membranes were embedded in a low-viscosity epoxy resin (Agar R1165) and sectioned with a Leica EM UC6 ultramicrotome. TEM samples of an isolated cyclodextrin film were prepared by dissolving the PAN support in DMSO and recovering the remaining $\beta$-CD film with a copper grid. XPS measurements were carried out in a Kratos Axis Ultra DLD spectrometer equipped with a monochromatic Al Ka X-ray source $(\mathrm{h} v=1486.6 \mathrm{eV})$ operating at $150 \mathrm{~W}$. The data was analysed with CasaXPS software. To characterise properties of the $\beta$-CD film alone, we isolated sufficient material by dissolving the PAN support of over 20 membranes with DMSO, transferred the remaining $\beta$ $\mathrm{CD}$ films to a water bath, washed them with methanol, and dried them at $60{ }^{\circ} \mathrm{C}$ under a vacuum. Thermal analysis of the isolated $\beta-\mathrm{CD}$ films was performed with a thermogravimetric analyser (TGA) Q5000 from TA Instruments under a $\mathrm{N}_{2}$ atmosphere from room temperature to $700{ }^{\circ} \mathrm{C}$ at a heating rate of $5{ }^{\circ} \mathrm{C} \mathrm{min}^{-1}$. Low-pressure $\mathrm{CO}_{2}$ sorption experiments on the isolated $\beta$-CD films were performed in a Micromeritics ASAP 2020. Samples were dried at $60{ }^{\circ} \mathrm{C}$ under a vacuum for 48 hours and degassed at $120{ }^{\circ} \mathrm{C}$ for 20 hours before measuring the $\mathrm{CO}_{2}$ sorption isotherm at $273 \mathrm{~K}$. Quantification of ferrocene methanol (Sigma-Aldrich) adsorbed by an isolated $\beta-C D$ film was done by immersing $5 \mathrm{~cm}^{2}$ 
of the film in a $200 \mathrm{ppm}$ ferrocene methanol in $\mathrm{MeOH}$ solution. This experiment was repeated three times. The ferrocene methanol solution was prepared by first dissolving ferrocene methanol in $1 \mathrm{ml}$ of acetone (Sigma-Aldrich) and then diluting the resulting solution with 199 $\mathrm{ml}$ of $\mathrm{MeOH}$. The concentration of ferrocene methanol was monitored by ultraviolet-visible (UV-vis) spectroscopy using a NanoDrop 2000c spectrophotometer.

Nanofiltration performance. It was evaluated in terms of permeance of water or organic solvents and rejection of $\mathrm{Na}_{2} \mathrm{SO}_{4}$ (Sigma-Aldrich) or dye molecules (methyl orange from Acros Organics, amido black 10B from MP Biomedicals, congo red from Sigma-Aldrich and brilliant blue R250 from Fisher Scientific) respectively. Nanofiltration experiments were carried out in a dead-end configuration using either Millipore stirred cells (model 8010, effective membrane area of $4.1 \mathrm{~cm}^{2}$ ) or high-pressure Sterlitech stainless steel cells (model HP4750, effective membrane area of $14.6 \mathrm{~cm}^{2}$ ). All experiments were done at room temperature and repeated three times. Data for consecutive solvent runs presented in Figure $3 \mathrm{c}$ were obtained using Sterlitech stainless steel cells with a driving force of 10 bar; the rest of the experiments were performed in Millipore stirred cells with a driving force of 4 bar. All membranes were preconditioned in the solvent to be measured by permeating it for at least one hour before taking any measurements. The solute concentration used in the tested solutions was 1000 ppm for $\mathrm{Na}_{2} \mathrm{SO}_{4}$ and $10 \mathrm{ppm}$ for the dye molecules. Analysis of the solute concentration was done with a NanoDrop UV- vis spectrophotometer for the dyes and with an Oakton pH/CON 510 conductivity meter for the salt.

Shape selective separations were tested with two pairs of molecules with similar molecular weights: PPIX (Sigma-Aldrich) and RB (Sigma-Aldrich); $\gamma$-CD and PEO 1500. For the first pair of molecules, PPIX was first dissolved in $1 \mathrm{ml}$ of DMSO and then diluted with $199 \mathrm{ml}$ of $\mathrm{MeOH}$ to a final concentration of $10 \mathrm{ppm}$ to prevent agglomeration of the dye molecules. This solution was then mixed in a 1:1 ratio with $10 \mathrm{ppm} \mathrm{RB}$ in $\mathrm{MeOH}$ solution to obtain the solution to be permeated through the $\beta$-CD membrane. The concentration of both molecules 
was monitored simultaneously via UV-vis spectroscopy. The other pair of molecules had to be tested separately because their signals overlapped in the gel-permeation chromatography (GPC) analysis used to monitor their concentration. The first solution tested was $1000 \mathrm{ppm}$ PEO 1500 in methanol, followed by pure $\mathrm{MeOH}$, and then $1000 \mathrm{ppm} \gamma-\mathrm{CD}$ in $\mathrm{MeOH}$ solution. Concentrations of $\gamma$-CD and PEO 1500 were measured with an Agilent system equipped with dual columns in series (PL Aquagel-OH $308 \mu \mathrm{m}$ and PL Aquagel-OH $408 \mu \mathrm{m}$ ) and a G1362A RID detector. Ultrapure Milli-Q water was used as the mobile phase with a flow rate of $1 \mathrm{ml} \mathrm{min}{ }^{-1}$.

\section{Supporting Information}

Supporting Information is available from the Wiley Online Library or from the author.

\section{Acknowledgements}

This research was supported by King Abdullah University of Science and Technology (KAUST). We are grateful to Kexin Yao for assisting with the $\mathrm{CO}_{2}$ adsorption experiments and to Mohamed Nejib Hedhili for performing the XPS analysis. We thank Rachid Sougrat, Stefan Chisca, Mahendra Kumar, Rahul Shevate, and Federico Pacheco for discussions.

Received: ((will be filled in by the editorial staff))

Revised: ((will be filled in by the editorial staff)) Published online: ((will be filled in by the editorial staff))

\section{References}

[1] P. Marchetti, M. F. Jimenez Solomon, G. Szekely, A.G. Livingston, Chem. Rev. 2014, $114,10735$.

[2] D. F. Sanders, Z. P. Smith, R. Guo, L. M. Robeson, J. E. McGrath, D. R. Paul, B. D. Freeman, Polymer 2013, 54, 4729.

[3] P. M. Budd, N. B. McKeown, D. Fritsch, J. Mater. Chem. 2005, 15, 1977.

[4] D. L. Gin, R. D. Noble, Science 2011, 332, 674. 
[5] H. B. Park, C. H. Jung, Y. M. Lee, A. J. Hill, S. J. Pas, S. T. Mudie, E. Van Wagner, B. D. Freeman, D. J. Cookson, Science 2007, 318, 254.[6] T. C. Merkel, B. D. Freeman, R. J. Spontak, Z. He, I. Pinnau, P. Meakin, A. J. Hill, Science 2002, 296, 519.

[7] N. Petzetakis, C. M. Doherty, A. W. Thornton, X. C. Chen, P. Cotanda, A. J. Hill, N. P. Balsara, Nat. Commun. 2015, 6.

[8] N. B. McKeown, P. M. Budd, Chem. Soc. Rev. 2006, 35, 675.

[9] J. R. Holst, A. Trewin, A. I. Cooper, Nat. Chem. 2010, 2, 915.

[10] H. Dodziuk, in Cyclodextrins and Their Complexes, Wiley-VCH Verlag GmbH \& Co. KGaA, Weinheim, Germany 2006, Ch. 1.

[11] E. M. M. Del Valle, Process Biochem. 2004, 39, 1033.

[12] E. Schneiderman, A. M. Stalcup, Chromatogr. B Biomed. Sci. Appl. 2000, 745, 83.

[13] N. Morin-Crini, G. Crini, Prog. Polym. Sci. 2013, 38, 344.

[14] A. Alsbaiee, B. J. Smith, L. Xiao, Y. Ling, D. E. Helbling, W. R. Dichtel, Nature 2016, $529,190$.

[15] P. Rölling, M. Lamers, C. Staudt, J. Memb. Sci. 2010, 362, 154.

[16] L. Yue, S. Wang, D. Zhou, H. Zhang, B. Li, L. Wu, Nat. Commun. 2016, 7, 10742.

[17] F. van de Manakker, T. Vermonden, C. F. van Nostrum, W. E. Hennink, Biomacromolecules 2009, 10, 3157.

[18] A. R. Khan, P. Forgo, K. J. Stine, V. T. D'Souza, Chem. Rev. 1998, 98, 1977.

[19] J. Hernandez-Pascacio, Á. Piñeiro, J. M. Ruso, N. Hassan, R. A. Campbell, J. Campos-Terán, M. Costas, Langmuir 2016, 32, 6682.

[20] G. González-Gaitano, P. Rodríguez, J. R. Isasi, M. Fuentes, G. Tardajos, M. Sánchez, J. Incl. Phenom. Macrocycl. Chem. 2002, 44, 101.

[21] P. W. Morgan, S. L. Kwolek, J. Polym. Sci. A Polym. Chem. 1996, 34, 531.

[22] F. Pacheco, R. Sougrat, M. Reinhard, J. O. Leckie, I. Pinnau, J. Memb. Sci. 2016, 501, 33. 
[23] S. Karan, Z. Jiang, A. G. Livingston, Science 2015, 348, 1347.

[24] E. Gaidamauskas, E. Norkus, E. Butkus, D. C. Crans, G. Grincienè, Carbohydr. Res. 2009, 344, 250 .

[25] T. Godjevargova, A. Dimov, J. Memb. Sci. 1992, 67, 283.

[26] M. F. Jimenez-Solomon, Q. Song, K. E. Jelfs, M. Munoz-Ibanez, A. G. Livingston, Nat. Mater. 2016, advance online publication.

[27] N. Joseph, J. Thomas, P. Ahmadiannamini, H. Van Gorp, R. Bernstein, S. De Feyter, M. Smet, W. Dehaen, R. Hoogenboom, I. F. J. Vankelecom, Adv. Funct. Mater. 2017, n/a.

[28] X. Peng, J. Jin, Y. Nakamura, T. Ohno, I. Ichinose, Nat. Nano. 2009, 4, 353.

[29] D. Wang, L.-J. Wan, C. Wang, C.-L. Bai, J. Phys. Chem. B 2002, 106, 4223.

[30] A. Harada, J. Li, M. Kamachi, Nature 1992, 356, 325.

[31] D.J. Cram, Science 1983, 219, 1177. 


\section{Table of contents}

Molecular-level design of a new type of filtration membrane made of crosslinked cyclodextrins - inexpensive macrocycles of glucose, shaped like hollow truncated cones. The channel-like cavities of cyclodextrins spawn numerous paths of defined aperture in the separation layer that can effectively discriminate between molecules. The transport of molecules through these membranes is highly shape-sensitive. In addition, the presence of hydrophobic (cavity) and hydrophilic (ester-crosslinked outer part) domains in these films results in high permeances for both polar and non-polar solvents.

\section{Keyword: Cyclodextrin membrane}

Luis Francisco Villalobos, Tiefan Huang and Klaus-Viktor Peinemann*

\section{Cyclodextrin films with fast solvent transport and shape-selective permeability}

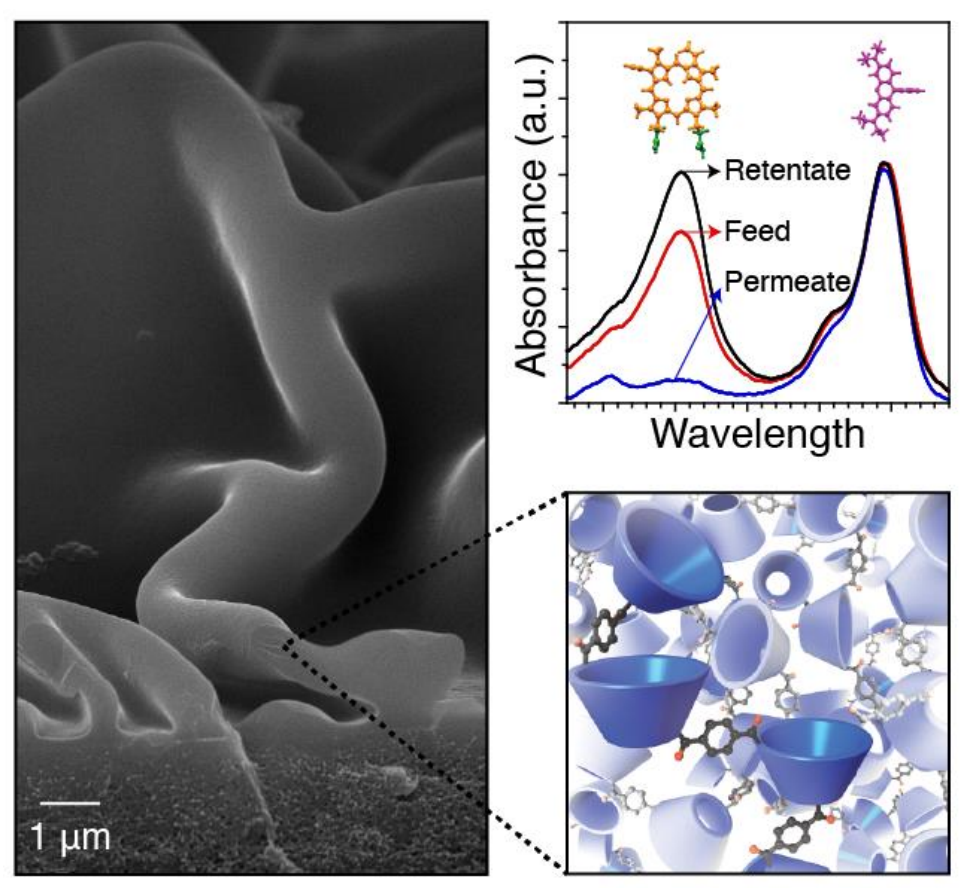

